DOI: https://doi.org/10.34069/AI/2021.43.07.11

How to Cite:

Popovych, I., Chervinskyi, A., Kazibekova, V., Chervinska, I., \& Machynska, N. (2021). Estudio empírico de la tipología de expectativas sociales del individuo. Amazonia Investiga, 10(43), 112-122. https://doi.org/10.34069/AI/2021.43.07.11

\title{
Estudio empírico de la tipología de expectativas sociales del individuo
}

\section{Empirical research of the typology of social expectations of the personality}

Received: June 12, 2021

Accepted: July 31, 2021

\author{
Written by: \\ Ihor Popovych ${ }^{39}$ \\ https://orcid.org/0000-0002-1663-111X \\ Andrii Chervinskyi ${ }^{40}$ \\ https://orcid.org/0000-0002-2128-196X \\ Viktoriia Kazibekova ${ }^{41}$ \\ https://orcid.org/0000-0001-8707-2514 \\ Inna Chervinska ${ }^{42}$ \\ https://orcid.org/0000-0003-0745-1413 \\ Natalia Machynska ${ }^{43}$ \\ https://orcid.org/0000-0003-0309-7074
}

\section{Resumen}

El propósito del estudio es determinar los parámetros de contenido psicológico y las propiedades de las expectativas sociales del individuo; investigar los tipos de expectativas sociales mediante el método de análisis de conglomerados; delinear el contenido sociopsicológico del fenómeno estudiado. Se determinan los niveles de expectativas sociales del individuo. Se establecen las propiedades predominantes de las expectativas en la situación actual de la actividad educativa y profesional. La agrupación del conjunto de datos estudiados está fundamentada e implementada metodológicamente. Métodos de investigación: análisis de contenido, pruebas con cuestionarios estandarizados, análisis factorial, análisis de conglomerados por k-medias. Se da la estructura de tipos de expectativas sociales del individuo. La clave en esta estructura es el tipo de expectativas sociales entendidas activamente $(\mathrm{n}=34 ; 43.55 \%)$. Los resultados obtenidos son importantes conocimientos sociopsicológicos que hacen un aporte científico al fenómeno estudiado. Creemos que la aplicación de los conocimientos adquiridos en la práctica

\begin{abstract}
The purpose of a research is to determine the psychological content parameters and properties of social expectations of the personality; research of types of social expectations by the method of cluster analysis; outlining the socio-psychological content of the studied phenomenon. The levels of social expectations of the personality are determined. The prevailing properties of expectations in the current situation of educational and professional activity are established. The clustering of the researched data set is methodologically substantiated and implemented. Research methods: content analysis, tests with standardized questionnaires, factor analysis, cluster analysis by k-means. The structure of types of the person's social expectations is given. The key in this structure is the actively meaningful type $(n=34 ; 43.55 \%)$ of social expectations. The received results are important socio-psychological knowledge that makes a scientific contribution to the researched phenomenon. We believe that the implementation of the received knowledge in practice will contribute to the effective organization of work in various spheres of human activity. The received knowledge is of scientific
\end{abstract}

\footnotetext{
${ }^{39}$ Doctor of Psychological Sciences, Full Professor of the Department of Psychology, Kherson State University, Kherson, Ukraine.

${ }^{40}$ Candidate of Historical Sciences, Associate Professor of the Department of Geography and Natural Sciences, Vasyl Stefanyk Precarpathian National University, Ivano-Frankivsk, Ukraine.

${ }^{41}$ Candidate of Psychological Sciences, Associate Professor of the Department of Psychology, Kherson State University, Kherson, Ukraine.

${ }^{42}$ Doctor of Pedagogical Sciences, Associate Professor Department of Pedagogy of Primary Education, Vasyl Stefanyk Precarpathian National University, Ivano-Frankivsk, Ukraine.

${ }^{43}$ Doctor of Pedagogical Sciences, Head of the Department of Primary and Preschool Education, Ivan Franko National University of Lviv, Lviv, Ukraine.
} 


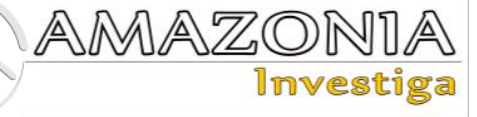

contribuirá a la organización eficaz del trabajo en las diversas esferas de la actividad humana. El conocimiento adquirido es de valor científico para todos los que estudian los patrones psicológicos de las expectativas sociales del individuo.

Palabras clave: expectativas, propiedades de expectativas, tipos de expectativas, interioridad, exterioridad.

\section{Introducción}

En la literatura científica existen muchos estudios científicos relacionados con la categoría de "expectativa" (Gustems-Carnicer et al., 2017; Kominis, 2007; Rotter, 1966). Se ha investigado la influencia de las expectativas sociopsicológicas en la efectividad de la interacción interpersonal, las características de las relaciones humanas (Popovych, 2007; 2014b). Por expectativas sociopsicológicas nos referimos a las orientaciones subjetivas de cualquier participante en la interacción interpersonal sobre cómo se evalúa a sí mismo y cómo lo evalúan otros participantes en la interacción y cuál comportamiento de los demás espera y los otros esperan de él. Por supuesto, las expectativas sociopsicológicas son una formación psicológica compleja, cuyo contenido depende del nivel de desarrollo de la persona, de sus características psicológicas individuales, de sus componentes de autoconciencia: autoestima, aspiraciones, reflexividad, así como formas integradas de autoconciencia tan complejas como la imagen de Yo y Yo - concepto (Popovych, 2019).

Un intento de una consideración fundamental de la categoría de expectativas sociopsicológicas merece atención. Se presta la debida atención a las funciones, los mecanismos psicológicos y el impacto de la sociedad en el comportamiento de la persona. Se concluye que las expectativas sociopsicológicas son una de las variedades que combinan las expectativas sociales. Estas variedades también incluyen expectativas de roles, expectativas normativas, expectativas colectivas y varios otros tipos. En nuestra opinión, éstas reflejan un aspecto separado del problema, completándose o negándose entre sí (Popovych, 2017b).

Las expectativas sociales son consideradas como una categoría científica global, un fenómeno complejo de la psicología social y la, de la personalidad, un mecanismo poderoso que tiene una influencia decisiva en el desarrollo y formación del individuo como sujeto. Así, las value for all who research the psychological patterns of social expectations of the personality.

Key words: expectations, properties of expectations, types of expectations internality, externality.

expectativas sociales no son solo las orientaciones subjetivas de cualquier participante en la interacción interpersonal, como se presenta en la definición de "expectativas sociopsicológicas" (Popovych, 2014a). Las expectativas sociales son un fenómeno complejo, con un contenido psicológico poco estudiado, que se forma, por un lado, como resultado de la influencia del espacio social, bajo la "presión de la sociedad", y por el otro, bajo la influencia de la personalidad psicológica individual, bajo la presión del "Yo".

Estas consideraciones científicas nos han llevado a estudiar la categoría de "expectativas sociales". Expectativas sociales son un fenómeno sociopsicológico complejo, que es un componente básico del sistema de regulación del comportamiento social, la interacción en grupos, comunidades y en la sociedad. Son los requisitos impuestos por un sujeto social - un individuo, un grupo, una comunidad, a otro sujeto social de comportamiento y viceversa (Popovych, 2017a).

Una persona siempre está conectada con las expectativas sociales, porque siempre quiere mirar hacia el futuro, prever, predecir el curso de los eventos. Todos esperan algo, ponen sus esperanzas y expectativas en algo. Cada momento de relaciones reacciones, conflictos y existencia humana en general está lleno de expectativas y esperanzas sociales.

El estudio de las expectativas sociales de un individuo nos obliga a determinar los parámetros psicológicos significativos, las propiedades de este fenómeno sociopsicológico y la identificación de tipos clave de expectativas sociales lo que nos permitirá comprender mejor la esencia de este fenómeno. Es sabido que el deseo de tipologizar los logros proporciona a cualquier investigación científica la sistematización, ordena y multiplica los conocimientos adquiridos. Nuestro estudio empírico destaca una de las opciones para 
estudiar los tipos de expectativas sociales del individuo.

Hipótesis. Suponemos que los hechos científicos obtenidos sobre los parámetros, propiedades y tipos de expectativas sociales del individuo son conocimientos sociopsicológicos importantes que hacen un aporte científico al fenómeno en estudio. La aplicación de los resultados de la investigación contribuirá a la organización eficaz del trabajo en diversos campos de actividad.

El propósito del estudio es determinar los parámetros de contenido psicológico y las propiedades de las expectativas sociales del individuo; investigar los tipos de expectativas sociales mediante el método de análisis de conglomerados; delinear el contenido sociopsicológico del fenómeno estudiado.

\section{Método}

La base metodológica de nuestro estudio empírico de la tipología de las expectativas sociales de un individuo es un algoritmo que refleja la secuencia de una serie de estudios probados utilizando herramientas de psicodiagnóstico relevantes. La metodología desarrollada por nosotros ha sido probada en la investigación de los procesos de adaptación (Arbeláez-Campillo et al., 2019; Blynova et al., 2020a; 2020d; Khmiliar et al., 2020), de la inteligencia emocional (Halian et al., 2020a; 2020b; Nosov et al., 2020a; 2020b) de los estados mentales motivacionales de los encuestados (Blynova et al., 2020b; 2020c; Popovych et al., 2020a; 2020b), de la actividad pedagógica (Griban et al., 2019; Ma et al., 2020; Miyer et al., 2021a; 2021b; Tsiuniak et al., 2020; Tsymbal et al., 2017; 2019) y otras actividades (Krupnyk \& Tkalenko, 2019; Popovych et al., 2021; Shkola et al. 2019; Zinchenko et al., 2019; 2020). La investigación científica propuesta es un estudio preempírico tangible de la tipología de expectativas sociales de una persona.

Participantes. Los estudiantes del segundo al cuarto año participaron en la investigación realizada en las instituciones de educación superior de Ucrania: la Universidad Estatal de Kherson [UEK], con 85 personas $(70.83 \%)$ y La Universidad Técnica Nacional de Jersón con 35 personas (29.17\%). La muestra total estuvo formada por 120 personas. La edad media de la muestra fue de 20.6 años ( $\mathrm{SD}=0.9$, de entre 18 y 25 años). La muestra incluyó al 70,83\% de las mujeres y al $29.17 \%$ de los hombres.
La investigación fue realizada de acuerdo con las normas éticas del comité sobre los derechos de los experimentos de Declaración de Helsinki (2013).

Organización del estudio. El corte empírico se realizó durante diciembre de 2019 y hasta febrero de 2020. Se obtuvo permiso y recomendaciones de las administraciones de facultades universitarias para la investigación empírica.

Procedimiento y instrumentos. Durante un semestre académico, se utilizaron instrumentos psicodiagnósticos que midieron los parámetros estudiados. Cuestionario "The level of social expectations" ("LSE”) (Popovych, 2017a). El índice de confiabilidad obtenido con las estadísticas de $\alpha$-Cronbach fue $\alpha=.759$. Cuestionario para el "Purpose in Life Test" ("PIL") (Leontyev, 2006). El índice de confiabilidad obtenido con las estadísticas de $\alpha$ Cronbach fue igual a $\alpha=.754$. Cuestionario "Nivel de declaraciones de personalidad" ("LPS") (Herbachevskyi, 1990). El índice de confiabilidad obtenido con las estadísticas de $\alpha$ Cronbach fue $\alpha=.767$. Cuestionario "The level of subjective control" ("LSC") (Rotter, 1966). El índice de confiabilidad obtenido con las estadísticas de $\alpha$-Cronbach fue $\alpha=.788$. Las respuestas fueron evaluadas con ayuda de una escala diferencial semántica bipolar similar. Por lo tanto, todos los indicadores de confiabilidad de $\alpha$-Cronbach estaban dentro de los límites de (.7) y niveles altos (.8).

Análisis estadístico. El procesamiento estadístico de los datos empíricos y la exposición gráfica de los resultados se llevaron a cabo utilizando los programas estadísticos "SPSS" v. 23.0 u otro nombre para PASW Statistics y "MS Excel". Los coeficientes de correlación de Spierman (rs) se utilizaron para encontrar y establecer las interrelaciones entre los indicadores obtenidos. La verificación de los parámetros de distribución normal fue hecha utilizando el criterio de recolección única de Kolmogorov-Smirnov $\chi$. Para establecer la homogeneidad de los datos empíricos se utilizó el análisis factorial. La definición de los tipos de expectativas sociales de los estudiantes jóvenes se realizó mediante análisis de conglomerados utilizando el método de k-medias. Se calcularon los valores medios aritméticos de los parámetros (M), la desviación media cuadrática (SD). Las diferencias entre los valores de los parámetros en el nivel $\mathrm{p} \leq .05$ se consideraron estadísticamente significativas. 


\section{AMAZONDA \\ 1nvestiga}

\section{Resultados y discusión}

1. Investigación de parámetros de contenido psicológico y propiedades de las expectativas sociales del individuo

En la etapa inicial de la investigación empírica, los parámetros significativos de las expectativas

Table 1.

Valores medios y desviaciones cuadráticas medias de las escalas de los parámetros de la personalidad $(n=120)$

\begin{tabular}{|c|c|c|}
\hline Escala & Media aritmética, $M$ & $\begin{array}{l}\text { Desviación de la media cuadrada, } \\
\text { SD }\end{array}$ \\
\hline \multicolumn{3}{|r|}{ ( } \\
\hline ESP & 65.40 & 12.59 \\
\hline CCPE & 16.69 & 3.05 \\
\hline AEPI & 14.77 & 1.70 \\
\hline REA & 34.78 & 8.58 \\
\hline \multicolumn{3}{|l|}{ "LSC" } \\
\hline IG & 199.01 & 19.03 \\
\hline IL & 54.59 & 7.26 \\
\hline IM & 50.90 & 7.59 \\
\hline IRF & 41.29 & 6.82 \\
\hline IRL & 36.88 & 4.90 \\
\hline ISE & 18.02 & 4.28 \\
\hline \multicolumn{3}{|l|}{ "PIL" } \\
\hline $\mathrm{OV}$ & 31.73 & 7.51 \\
\hline $\mathrm{P}$ & 29.55 & 5.27 \\
\hline $\mathrm{R}$ & 25.20 & 4.74 \\
\hline LCI & 20.97 & 4.44 \\
\hline LCE & 29.83 & 4.58 \\
\hline SGL & 101.10 & 13.90 \\
\hline \multicolumn{3}{|l|}{ "LPS" } \\
\hline MI & 12.76 & 3.11 \\
\hline MC & 15.60 & 2.39 \\
\hline ME & 11.50 & 3.85 \\
\hline MC & 11.90 & 3.12 \\
\hline MCA & 12.90 & 3.01 \\
\hline MA & 14.20 & 3.09 \\
\hline IR & 8.94 & 2.07 \\
\hline $\mathrm{CT}$ & 5.67 & 2.01 \\
\hline $\mathrm{EV}$ & 12.79 & 3.91 \\
\hline ENRA & 10.03 & 2.04 \\
\hline $\mathrm{EP}$ & 13.19 & 3.19 \\
\hline NPME & 14.24 & 2.03 \\
\hline $\mathrm{RE}$ & 9.52 & 2.95 \\
\hline $\mathrm{RR}$ & 13.50 & 2.92 \\
\hline I & 13.39 & 2.99 \\
\hline
\end{tabular}

Se encontró que el estado predominante de interioridad de expectativas fue en $20.01 \%$, el estado de externalidad de expectativas se vio en $22.93 \%$, la actividad de expectativas se observó en $21.03 \%$ de los encuestados, el estado de expectativas pasivas, en $23.17 \%$, de expectativas abiertas, en $39.13 \%$, el estado mental de expectativas cerradas en $25.01 \%$, de expectativas adecuadas, en $41.26 \%$ y de expectativas inadecuadas, en $22.17 \%$ de los sujetos. Hubo manifestaciones de polaridad de expectativas en $12.3 \%$ y ambivalencia de expectativas en $4,0 \%$. sociales de los encuestados se determinaron utilizando los métodos de prueba estandarizados anteriores. Se evaluaron los resultados obtenidos de los parámetros estudiados, enfocándose en las escalas de mínimo (min), máximo (max), valor medio aritmético (M) y desviación estándar (DE), presentados en la Tabla 1. 
Durante la investigación, analizamos una gran cantidad de discursos creados por las personas estudios. Se han analizado los textos de situaciones sociopsicológicas especialmente organizadas, de la práctica cotidiana, en particular las conversaciones de los participantes de interacción interpersonal que han tenido lugar en situaciones cotidianas. Es obvio que el análisis del discurso se ha implementado en el contexto del estudio del problema de las expectativas sociales del individuo. El análisis del discurso nos devuelve al diagnóstico psicolingüístico del espacio cognitivo individual que es un conjunto único de todos los conocimientos e ideas de una persona como personalidad integral 3. Se realizó un análisis estadístico adicional de acuerdo con los datos de solo aquellos encuestados cuyas respuestas tenían una descripción completa de las expectativas sociales y del comportamiento en la situación actual $(n=120)$.

Creemos que el conjunto propuesto de parámetros psicológicos es metodológicamente sólido, reflejando el tema de investigación de los parámetros de contenido psicológico y las propiedades de las expectativas sociales de la juventud estudiantil. Pasemos a la siguiente parte del estudio empírico: la agrupación de tipos de expectativas sociales de la persona.

\section{Investigación y característica de los tipos de expectativas sociales de la persona}

El análisis de conglomerados se utilizó por primera vez como método en la ciencia sociológica. El nombre "análisis de conglomerados" proviene del inglés clúster - un racimo, un conglomerado, un clúster. El investigador R. Tryon fue el primero en definir el análisis de conglomerados: reveló su esencia y presentó el método de aplicación (Tryon, 1939). El análisis de conglomerados es un procedimiento estadístico que realiza una clasificación multidimensional de datos que contienen información sobre una muestra de objetos, es decir, organiza los objetos en grupos relativamente homogéneos (Lloyd, 1957). El análisis de conglomerados por el método de kmedias consiste en combinar los parámetros de factores individuales en grupos estadísticamente homogéneos: conglomerados. El propósito del método es dividir $\mathrm{n}$ mediciones en $\mathrm{k}$ grupos de modo que cada dimensión de las personas en estudio pertenezca al grupo con el valor promedio más cercano. El método se basa en minimizar la suma de los cuadrados de las distancias entre cada observación y el centro de su grupo, es decir, la función (ver Fórmula 1):

$$
\sum_{i=1}^{N} d\left(x_{i}, m_{j}\left(x_{i}\right)\right)^{2}
$$

donde $\mathrm{d}$-métrica; $\mathrm{x}_{\mathrm{i}}$ - i-ésimo objeto de datos; $\mathrm{m}_{\mathrm{j}}\left(\mathrm{x}_{\mathrm{i}}\right)$ - centro del clúster, al que en la j-ésima iteración se le asigna el elemento $\mathrm{x}_{\mathrm{i}}$.

La simplicidad, la velocidad de ejecución y la conveniencia de la aplicación para la agrupación de un gran número de observaciones $(\mathrm{n}=120)$ nos llevaron a detener nuestra elección en el análisis de agrupaciones por el método de k-medias al determinar los tipos de expectativas de la muestra. Después de analizar una gran variedad de datos, parece lógico combinar los parámetros estudiados de las expectativas sociales del individuo en conjuntos organizados de indicadores estudiados, es decir, taxonomías visuales. Una variante adecuada de esta taxonomía es el análisis de conglomerados por el método de k-medias (k-means clustering), que consiste en combinar los parámetros de los factores individuales en grupos estadísticamente homogéneos - conglomerados.

El análisis de conglomerados es un método indispensable para clasificar una gran variedad de información en grupos que son adecuados para un análisis posterior. El análisis de conglomerados por el método de k-medias proporciona la solución significativa-más probable. Implementamos el análisis de conglomerados en varias etapas. Los datos empíricos se obtuvieron en la etapa preparatoria $(n=120)$. En la segunda etapa, se prepararon los datos para para su agrupación. La elección de los indicadores estudiados, mediante los cuales se realizará la agrupación, es de gran importancia metodológica. Consideramos que la lista seleccionada es metodológicamente sólida y la que define de manera más completa las características del contenido del tema de investigación. Por supuesto, el contenido sociopsicológico establecido de las expectativas dependerá de ciertas características. Usamos el nivel de expectativas sociales como característica principal que es básica y combina dos dimensiones psicológicas: personal y social. Así, se seleccionan cuatro rasgos que caracterizan los determinantes sociopsicológicos de las expectativas personales, y los cuatro rasgos siguientes caracterizan a las expectativas sociales como un factor importante en la construcción de la realidad social por parte del individuo. Esta metodología de análisis de conglomerados permitió tener en cuenta la misma cantidad de parámetros de las dimensiones personal y social del estudio de las expectativas sociales del individuo. A continuación, calculamos la visualización objetiva de características 


\section{AMAZONDA \\ 1nvestiga}

significativas en los siguientes grupos. Por supuesto, partimos de los niveles de expectativas sociales del individuo como dimensión básica. Solo en la tercera etapa, nos convencimos de que lo más conveniente en nuestra versión es aplicar el análisis de conglomerados mediante el método de k-medias. Después de eso, el siguiente paso fue la distribución de $\mathrm{n}$ mediciones de los sujetos estudiados en $\mathrm{k}$ grupos de modo que cada medición del sujeto perteneciera al grupo con el valor promedio más cercano. Tengamos en cuenta que uno de los requisitos que debe cumplir el investigador es el número mínimo de mediciones de los encuestados en el grupo, en nuestra versión $n \geq 18$. Un requisito igualmente importante fue mantener la aleatoriedad y la homogeneidad de la muestra. Usando "SPSS" v. 23.0, se estableció la homogeneidad debido a una disminución en la proporcionalidad mediante análisis factorial. Se establece que el criterio de selectividad adecuada de KaiserMayer-Olkin (KMO) es igual a .759 (se debe utilizar el análisis de conglomerados cuando éste está por encima de .5), lo cual es un resultado positivo. El criterio de esfericidad de Bartlett es significativo $(\mathrm{p}<.001)$. Este hecho nos permitió aplicar razonablemente el análisis de conglomerados. Además, el número óptimo de grupos se estableció mediante el método de pruebas: cinco. El primer objeto de la agrupación es el parámetro básico de nuestro estudio: el nivel de expectativas sociales (ESP), lo consideramos el centro del primer grupo, el segundo, del segundo, etc. Creemos que está metodológicamente justificado iniciar la investigación de clúster y establecer el inicio de la agrupación a partir del nivel de expectativas sociales. Mostramos los centros iniciales de los grupos en la tabla 2 .

Table 2.

Centros iniciales de clústeres $(n=120)$

\begin{tabular}{llllll}
\hline Parámetros & $\begin{array}{l}\text { Grupo 1 } \\
(\mathbf{n = 1 9 )}\end{array}$ & $\begin{array}{l}\text { Grupo 2 } \\
(\mathbf{n = 2 0})\end{array}$ & $\begin{array}{l}\text { Grupo 3 } \\
(\mathbf{n = 2 2})\end{array}$ & $\begin{array}{l}\text { Grupo 4 } \\
(\mathbf{n = 3 4 )}\end{array}$ & $\begin{array}{l}\text { Grupo 5 } \\
(\mathbf{n = 2 5})\end{array}$ \\
\hline ESP & 92 & 83 & 82 & 71 & 40 \\
IG & 245 & 135 & 212 & 208 & 152 \\
SGL & 124 & 124 & 40 & 154 & 61 \\
ENRA & 19 & 14 & 22 & 18 & 10 \\
NPME & 8 & 14 & 14 & .64 & 4 \\
IM & .80 & .64 & .68 & .56 & .14 \\
IRF & .89 & .66 & 1.0 & 1.08 & .16 \\
IRL & 0.9 & 1.2 & .15 & .55 & .61 \\
ISE & .17 & .72 & & & .41 \\
\hline
\end{tabular}

Una característica importante del análisis de conglomerados de k-medias es que, como resultado de la optimización, las diferencias entre los objetos del mismo conglomerado se vuelven insignificantes y las, de diferentes conglomerados se hacen significativas. La determinación de los centros iniciales de los conglomerados es una condición necesaria para establecer diferencias mínimas entre los indicadores estudiados de un conglomerado. Mostramos los centros finales de los grupos en la tabla 3.

Table 3.

Centros finales de clústeres $(n=120)$

\begin{tabular}{llllll}
\hline Parámetros & $\begin{array}{l}\text { Grupo 1 } \\
(\mathbf{n = 1 9 )}\end{array}$ & $\begin{array}{l}\text { Grupo 2 } \\
(\mathbf{n = 2 0})\end{array}$ & $\begin{array}{l}\text { Grupo 3 } \\
(\mathbf{n = 2 2})\end{array}$ & $\begin{array}{l}\text { Grupo 4 } \\
(\mathbf{n = 3 4})\end{array}$ & $\begin{array}{l}\text { Grupo 5 } \\
(\mathbf{n = 2 5})\end{array}$ \\
\hline ESP & 85 & 73 & 72 & 74 & 64 \\
IG & 235 & 172 & 208 & 203 & 180 \\
SGL & 117 & 108 & 88 & 114 & 84 \\
ENRA & 15 & 15 & 16 & 15 & 12 \\
NPME & 10 & 11 & 10 & 11 & 10 \\
IM & .74 & .57 & .59 & .61 & .47 \\
IRF & .70 & .57 & .57 & .58 & .46 \\
IRL & .71 & .66 & .68 & .71 & .61 \\
ISE & .36 & .34 & .33 & .35 & .35 \\
\hline
\end{tabular}


El establecimiento de los centros finales de los conglomerados muestra los límites del conjunto de indicadores estudiados, referidos a un conglomerado en particular. Consideramos necesario caracterizar los clústeres obtenidos. Estos grupos representan una taxonomía visual. La secuencia de los indicadores estudiados indica que después del nivel de expectativas sociales la prioridad para nosotros es una dimensión vertical - determinantes sociopsicológicos de las expectativas personales, y luego están los indicadores estudiados de expectativas sociales como factor de construcción de la realidad social. El análisis de los resultados obtenidos se realizará en la secuencia especificada teniendo en cuenta las características socio personales e interpersonales. Damos una descripción de los conglomerados obtenidos, en base a los valores medios y desviaciones estándar de los parámetros.

Clúster 1 (tipo I) es el más pequeño en número $(n=19)$, pero cumple con los requisitos del número mínimo de opciones. Los sujetos de este tipo están dotados de un alto nivel de expectativas sociales, un alto nivel de control subjetivo, un alto nivel de significación general de la vida, un alto nivel de movilización planificada de esfuerzos, un nivel medio de resultados esperados, un nivel medio de interioridad, un nivel medio de actividad, un alto nivel de apertura. Las características importantes de este tipo de expectativas son aquellas que lo relacionan con un alto nivel de expectativas sociales, y en cuanto a su contenido, las expectativas de dichos encuestados son internas, activas y abiertas.

Clúster 2 (tipo II) también tiene un número pequeño de personas $(n=20)$. Los sujetos de este tipo están dotados de un nivel medio de expectativas sociales, un bajo nivel de control subjetivo, indicadores de niveles medios y altos de significación general de la vida, nivel medio de movilización planificada de esfuerzos, indicadores de niveles medios y altos de resultados esperados, un bajo nivel de interioridad y de actividad, de la apertura, indicadores de promedio y altos niveles de coeficiente de expectativa.

Clúster 3 (tipo III) combina el grupo medio de sujetos con el número total $(n=22)$. Sujetos de este tipo están dotados de un nivel medio de expectativas sociales, indicadores de niveles medio y alto de control subjetivo, un bajo nivel de significación general de la vida, indicadores de niveles medios y altos de movilización planificada de esfuerzos, indicadores de niveles medio y alto de resultados esperados, de una baja interioridad y actividad, de la apertura, un nivel medio de expectativas.

Clúster 4 (tipo IV) es el más grande en número $(n=34)$. Los encuestados de este tipo también están dotados de un nivel medio de expectativas sociales, tienen un nivel medio de control subjetivo, indicadores de niveles medios y altos de significación general de la vida, indicadores de niveles bajos y medios de movilización planificada de esfuerzos, un nivel medio y alto del resultado esperado, un nivel bajo de interioridad y de actividad, de la apertura, indicadores de un nivel medio del coeficiente de expectativas.

Clúster 5 (tipo V) El grupo 5 (tipo V) reúne a un número significativo de encuestados con un número total $(\mathrm{n}=25)$. Los sujetos de este tipo tienen un nivel bajo de expectativas sociales, niveles bajo y medio de control subjetivo, un nivel bajo de significación general de la vida, indicadores de niveles bajos y medios de movilización planificada de esfuerzos, un nivel bajo de resultados esperados, externalidad, pasividad, introversión, indicadores del nivel medio del coeficiente de expectativas. Las características importantes de este tipo de expectativas son aquellas que lo atribuyen a un bajo nivel de expectativas sociales, tienen un bajo nivel de resultados esperados. En cuanto a sus características significativas, las expectativas de dichos sujetos son externas, pasivas y cerradas. Así, la agrupación por el método de kmedias mostró la integración original de la muestra en cinco agrupaciones y permitió hablar de cinco tipos de expectativas sociales del individuo.

Tipo realista (RL). Alto nivel de expectativas sociales de los encuestados con altos indicadores de control del subjetivo y significado general de la vida. Tienen un alto nivel de movilización planificada de esfuerzos y un nivel medio de los resultados esperados. Las expectativas están dotadas de altos índices de interioridad, actividad y apertura.

Tipo de pensamiento pasivo (PP). Nivel medio de expectativas sociales de los encuestados con indicadores bajos de control subjetivo e indicadores medios y altos de significado general de la vida. Tienen un nivel medio de movilización planificada de esfuerzos y niveles medios y altos de resultados esperados. Las expectativas son internas, activas y abiertas. 


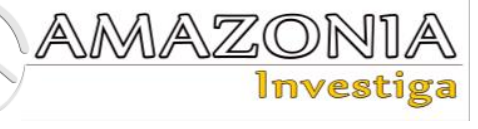

Tipo moderado (MD). El nivel medio de expectativas sociales de los sujetos con indicadores de niveles medio y alto de control subjetivo y un nivel bajo de significado general de la vida. Los sujetos están dotados de indicadores de niveles medios y altos de movilización planificada de esfuerzos y resultados esperados. Las expectativas son internas, activas y abiertas.

Tipo de pensamiento activo (PA). El nivel medio de expectativas sociales de los sujetos con indicadores del nivel medio de control subjetivo y los niveles medio y alto de significado general de la vida. Tienen indicadores de niveles bajos y medios de movilización de esfuerzos planificada, y niveles medios y altos de resultados esperados.
Tipo poco realista (PR). Bajo nivel de expectativas sociales de los sujetos con niveles bajo y medio de control subjetivo, y bajo nivel de significado general de la vida. Tienen indicadores de niveles bajos y medios de movilización de esfuerzos planificada y un nivel bajo de resultados esperados. Las expectativas sociales de los sujetos son externas, pasivas y cerradas.

Los complejos obtenidos y analizados de los indicadores estudiados o taxonomías o clasificaciones visuales (tipologías) permiten afirmar la presencia de cinco tipos de expectativas sociales de un individuo, e indican la legitimidad de tal distribución. Mostremos en la Fig. 1 la estructura de tipos de expectativas sociales del individuo.



Figura 1. La estructura de tipos de expectativas sociales del individuo

Nota: RL - tipo realista; PP - tipo de pensamiento pasivo; MD - tipo moderado; PA - tipo de pensamiento activo; PR es un tipo poco realista.

Los primeros cuatro tipos de expectativas están dotados de propiedades internas, activas $\mathrm{y}$ abiertas. PP, MD, PA es una especie de la descripción en detalle del nivel medio de expectativas sociales con opciones para características significativas. Los indicadores bastante altos de PR son alarmantes, ellos se caracterizan por indicadores de bajos niveles de expectativas sociales, control subjetivo, significado general de la vida en combinación con externalidad, pasividad y aislamiento de las propiedades de las expectativas. La distribución del coeficiente de expectativa para todos los tipos es uniforme. Explicamos los indicadores predominantes del tipo $\mathrm{V}$ en los encuestados principalmente por determinantes socio personales que inciden en el tipo de expectativas, y solo entonces por la presión social y otras influencias sociales.

\section{Conclusiones}

1. Se define, que los determinantes sociopersonales, el nivel de autoorganización de las actividades educativas y profesionales y el espacio sociocultural de las instituciones de educación superior inciden de manera decisiva en los parámetros de contenido psicológico, propiedades y tipos de expectativas sociales del individuo, en una versión particular de la juventud estudiantil.

2. Los resultados empíricos y la justificación de la medición de expectativas mostraron la legitimidad de los indicadores de expectativas sociales seleccionados. La secuencia de agrupamiento de los parámetros que caracterizan los determinantes sociopsicológicos de las expectativas personales con los parámetros 
que determinan la dimensión social de las expectativas de los encuestados, brindó una oportunidad para establecer vínculos causales entre tipos de expectativas, lo que permitió fundamentar la relación entre las dimensiones personal y social de las expectativas de los estudiantes jóvenes y da motivos para afirmar que las hipótesis fueron confirmadas.

3. Afirmamos que se han obtenido importantes conocimientos sociopsicológicos, que hacen un aporte científico al fenómeno estudiado. Creemos que la aplicación de los resultados del estudio contribuirá a la organización eficaz del trabajo en diversas áreas de la actividad humana.

\section{Referencias}

Arbeláez-Campillo, D. F., Rojas-Bahamón, M. J., \& Arbeláez-Encarnación, T. F. (2019). Apuntes para el debate de las categorías ciudadanía universal, derechos humanos y globalización // Notes for the debate of the categories universal citizenship, human rights and globalization. Cuestiones Políticas, 34(61), 139-161, https://produccioncientificaluz.org/index.php/cu estiones/article/view/30192

Blynova, O., Chervinska, I., Kazibekova, V., Bokshan, H., Yakovleva, S., Zaverukha, O., \& Popovych I. (2020a). Social and Psychological Manifestations of Professional Identity Crisis of Labor Migrants. Revista Inclusiones, 7(3), 93-105,

http://www.revistainclusiones.org/index.php/inc lu/article/view/1318

Blynova, O., Lappo, V., Kalenchuk, V., Agarkov, O., Shramko, I., Lymarenko, L., \& Popovych, I. (2020b). Corporate Culture of a Higher Education Institution as a Factor in Forming Students' Professional Identity. Revista Inclusiones, 7(Especial), 481-496, http://www.revistainclusiones.org/index.php/inc lu/article/view/1305

Blynova, O., Moiseienko, V., Los, O., Burlakova, I., Yevdokimova, O., Toba, M., \& Popovych, I. (2020c). Assertiveness as a Factor of Students' Choice of Behavior Strategies in Social Interaction. Revista Inclusiones, 7(4), 259-272,

http://www.revistainclusiones.org/index.php/inc lu/article/view/1551

Blynova, O., Popovych, I., Semenova, N., Kashyrina, Ye., Ursulenko, O., \& Kononenko, O. (2020d). Personality Factors of Choosing Adaptation Strategies in a Different Cultural Environment by Labor Migrants from Ukraine. Revista Amazonia Investiga, 9(32), 45-54, http://dx.doi.org/10.34069/AI/2020.32.08.5
Griban, G., Prontenko, K., Yavorska, T., Bezpaliy, S., Bublei, T., Marushchak, M., Pustoliakova, L., Andreychuk, V., Tkachenko, P., Zhukovskyi, Ye., Baldetskiy, A., \& Bloshchynskyi, I. (2019). Non-traditional means of physical training in middle school physical education classes. International Journal of Applied Exercise Physiology, 8(3.1), 224-32. DOI: 10.26655/IJAEP.2019.10.1

Gustems-Carnicer, J., Calderon, C., \& Forn Santacana, M. (2017). Psychometric properties of the Life Orientation Test (LOT-R) and its relationship with psychological wellbeing and academic progress in college students. Revista Latinoamericana de Psicología, 49, 19-27. DOI: 10.1016/j.rlp.2016.05.001

Halian, I. M., Halian, O. I., Gusak, L. Ye., Bokshan, H. I., \& Popovych, I. S. (2020a). Communicative Competence in Training Future Language and Literature Teachers. Revista Amazonia Investiga, 9(29), 530-541, https://amazoniainvestiga.info/index.php/amazo nia/article/view/1417

Halian, I., Machynska, N., Lozynska, S., Nos, L., Derkach, Yu., Prots, M., \& Popovych, I. (2020b). Tolerance of uncertainty as a component of the process of life-creation of future educators. Revista Inclusiones, 7(Especial), 512-528, http://www.revistainclusiones.org/index.php/inc lu/article/view/1307

Herbachevskyi, K. (1990). Psychological diagnostics. Methodology: the level of personality claims. St. Petersburg: Rech.

Khmiliar, O., Popovych, I., Hrys, A., Pavliuk, M., Zavatska, N., Lytvynenko, O., \& Blynova, O. (2020). Spatial Regulation of Personality Behavior in the Conditions of Progression of the COVID-19 Pandemic. Revista Inclusiones, 7(Especial), 289-306, http://www.revistainclusiones.org/index.php/inc lu/article/view/1760

Kominis, G. (2007). The expectancy-valence the or y revisited: developing an extended model of managerial motivation. Management Accounting Research, 18(1), 49-75, DOI: 10.1016/j.mar.2006.10.002

Krupnyk, I. R., \& Tkalenko, N. V. (2019). Manipulative Behavior in the Professional Activities of Office Staff. Insight: the psychological dimensions of society, 1, 96-101. DOI: $10.32999 / 2663-970 X / 2019-1-15$

Leontyev, D. (2006). Test of life-meaning full orientations ('LMO'). Psychodiagnostic series. Moscow: Smysl.

Lloyd, S. (1957). Least square quantization in PCM's. New York: Bell Telephone Laboratories Paper.

Ma, F., Shevchenko, R. P., \& Karhina, N. V. (2020). Student you three presentation of 


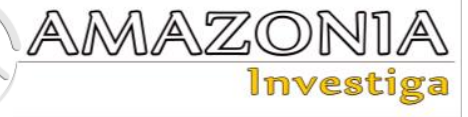

psychological well-being: results of content analysis of works. Insight: the psychological dimensions of society, 3, 44-55. DOI: $10.32999 / 2663-970 X / 2020-3-3$

Miyer, T., Holodiuk, L., Omelchuk, S., Savosh, V., Bondarenko, H., Romanenko, L., \& Romanenko, K. (2021a). An Overview of the Continuous Education System Components in Dimensions "Umwelt", "Mitwelt" and "Eigenwelt". AD ALTA: Journal of Interdisciplinary Research, 11(1), 52-56.

Miyer, T., Holodiuk, L., Omelchuk, S., Savosh, V., Bondarenko, H., Rudenko, N., \& Shpitsa, R. (2021b). ICT as a Means of Implementing thematic FIN-modeling in the organization of training in institutions of higher pedagogical and adult education. AD ALTA: Journal of Interdisciplinary Research, 11(1), 26-32.

Nosov, P., Palamarchuk, I., Zinchenko, S., Popovych, I., Nahrybelnyi, Y., \& Nosova, H. (2020). Development of means for experimental identification of navigator attention in ergatic systems of maritime transport. Bulletin of the University of Karaganda - Physics, 1(97), 58-69, https://doi.org/10.31489/2020Ph1/58-69

Nosov, P. S., Zinchenko, S. M. Popovych, I. S. Ben, A. P., Nahrybelnyi, Y. A., \& Mateichuk, V. M. (2020). Diagnostic system of perception of navigation danger when implementation complicated maneuvers. Electronics, Computer Science, Control, 1, 146-161. DOI: 10.15588/1607-3274-2020-1-15 Popovych, I., Arbeláez-Campillo, D. F., Rojas-Bahamón, M. J., Burlakova, I., Kobets, V., \& Bokshan, H. (2021). Perspectivas temporales en las actividades profesionales de especialistas en el área económica. Cuestiones Políticas, 39(69), 424-445,

https://doi.org/10.46398/cuestpol.3969.27

Popovych, I., Lymarenko, L., Tereshenko, N., Kornisheva, T., Yevdokimova, O., Koverznieva, A., \& Aleksieieva, M. (2020a). Research on the Effectiveness of Training Technologies' Implementation in Student Theater. Revista Inclusiones, 7(2), 104-121, http://www.revistainclusiones.org/index.php/inc lu/article/view/261

Popovych, I. S. (2017a). Psychological dimensions of social expectations of personality. Kherson: KTPH.

Popovych, I. S. (2017b). Psychology of social expectations of personality. Extended abstract of Doctor's thesis. Severodonetsk: Volodymyr Dahl East-Ukrainian National University.

Popovych, I. S. (2019). Psychology of Social Expectations of Personality: Methodology, Theory and Practice. Kherson: OLDI-PLUS.
Popovych, I. S. (2007). Social and psychological expectations in interpersonal interaction in the groups of cadets of higher educational institutions of The Ministry of Internal Affairs of Ukraine. Extended abstract of candidate's thesis. Kyiv: G. S. Kostiuk Institute of Psychology, NAPS of Ukraine.

Popovych, I. S. (2014a). Social expectations in the theories of motivation of personality. Philosophy. Psychology. Sociology, 4(20), 53-63.

Popovych, I. S. (2014b). Typological peculiarities of expectations. Scientific Herald of Kherson State University, 1, 64-70.

Popovych, I., Zhigarenko, I., Losiyevska, O., Dovbenko, S., Kashyrina, Ye., Shevchenko, R., \& Piletska, L. (2020b). Research of Achievement Motivation's Impaction the Career Orientations of Future Managers of Organization. Revista Inclusiones, 7(Especial), 247-263, http://www.revistainclusiones.org/index.php/inc lu/article/view/1231

Rotter, J. (1966). Generalized expectancies for internal versus external control of reinforcement. Psychological Monographs: General and Applied, 80(1), 1-28.

Shkola, O., Griban, G., Prontenko, K., Fomenko, O., Zhamardiy, V., Bondarenko, V., Bezpaliy, S., Andreychuk, V., Tkachenko, P., Zhukovskyi, Ye., Novitska, I., Bloshchynskyi, I. (2019). Formation of valuable orientations in youth during physical training. International Journal of Applied Exercise Physiology, 8(3.1), 264-272. DOI: 10.26655/IJAEP.2019.10.1

Tryon, R. C. (1939). Cluster analysis. London: lithoprinters and publishers.

Tsiuniak, O., Pyslar, A., Lialiuk, G., Bondarenko, V., Kovtun, O., Los, O., \& Popovych, I. (2020). Research of interdependence of variables and factor structure of masters' readiness for innovative pedagogical activity. Revista Inclusiones, 7(3), 427-452, http://www.revistainclusiones.org/index.php/inc lu/article/view/1645

Tsymbal, S. V. (2019). Enhancing students' confidence and motivation in learning English with the use of online game training sessions. Information Technologies and Learning Tools, 71(3), 227-235.

Tsymbal, S. V. (2017). Overcoming language anxiety among the English language learners: psycho-pedagogical aspect. Science and Education, 7, 102-106.

World Medical Association Declaration of Helsinki. (2013). Ethical principles for medical research involving human subjects, 310(20), 2191-4,

https://doi.org/10.1001/jama.2013.281053 
Zinchenko, S. M., Ben, A. P., Nosov, P. S., Popovych, I. S., Mamenko, P. P., \& Mateychuk, V. M. (2020). Improving the accuracy and reliability of automatic vessel moution control systems. Radio Electronics, Computer Science, Control, 2, 183-195. DOI: 10.15588/1607-3274-2020-2-19
Zinchenko, S., Nosov, P., Mateichuk, V., Mamenko, P., Popovych, I., \& Grosheva, O. (2019). Automatic collision avoidance system with many targets, including maneuvering ones. Bulletin of the University of Karaganda Physics, 4(96), $69-79$, https://doi.org/10.31489/2019Ph4/69-79 